\title{
Intervening in status hierarchies to disrupt inequity
}

\author{
Lily Payne and Paul Hutchison
Education Department, Grinnell College, 1120 Park St., Grinnell, IA, 50112
}

In order to support equitable science education, it is vital to understand how in/equity arises in a classroom. Because power dynamics are inextricably linked to in/equity, we investigated the way in which student status hierarchies affect in/equity in small student groups. We conducted a mixed methods case study analysis of a small group of rising college freshmen participating in a summer science program for students with traditionally under-represented identities. Our case study covers six sessions of one small group. The analysis shows that overall the established status hierarchy of the group contributed to a pattern of inequitable student interactions. The only significant deviation from this pattern occurred when an instructor intervention disrupted the status hierarchy of the students, which resulted in greater equity for one session. These results suggest that instructor interventions can have a profound impact on student power dynamics and the quality of small group interactions.

\section{INTRODUCTION}

Inequity has long been an issue in science education. Limited representation of people of color, women, first generation students, etc. indicate that certain identities are marginalized in science classrooms [1]. In order to remedy inequity in science, we must come to understand how and why inequity manifests in science classrooms.

Equity can be defined as the fair distribution of opportunities to learn, which involves both one's access to content as well as one's access to positional identities as a knower and a doer [2]. Researchers have studied equity in education in order to understand achievement, fairness, and opportunity in educational contexts. We sought to build on this literature by exploring inequity in collaborative small group interactions.

Collaborative learning is an instructional method utilized in many science classrooms in which students work together to collaboratively solve a problem. This method is meant to give students opportunities to collaborate with, learn from, and teach their peers. While collaborative learning is intended to foster productive student collaborations, in practice it is not uncommon for group work to create or exacerbate inequity between students [3].

Inequity in small groups can arise when status and power are unevenly distributed within a group, resulting in some students being positioned as more competent or authoritative than others. [4] This social positioning can diminish students' opportunities to learn by limiting students' access to content and access to positional identities as knowers and doers [2]. It is therefore vital for educators to learn how to support productive and equitable student interactions in cooperative learning.

This paper attempts to analyze the relationship between social positioning and equity in student interactions. We report the findings of a case study on the negotiation of status and its effect on in/equity in a small student group in a collegiate science setting. We argue that patterns of inequity can arise as a result of status hierarchies in cooperative learning groups. We find evidence that these status hierarchies can be interrupted in order to disrupt inequity and promote more equitable student interactions.

\section{METHODOLOGY}

\section{A. Data Collection}

We analyzed the interactions of a single collaborative group of students in the IMPRESS program, a preorientation science program at the Rochester Institute of Technology designed to improve the persistence of science interested students among deaf/hard of hearing students and first generation college students [5].

The group we focus on was selected for analysis because it contains a lot of what appears to be typically inequitable interactions among the members. There is a single group member who dominates much of the group activity and group decision making. Other group members, one in particular, make repeated efforts to contribute and are rebuffed. There is occasional tension in the group as a result of these kinds of interactions. The group consists of four students: Justin, a white man; Jessica, a deaf/hard of hearing white woman; Brittany, a Native American/Latina woman; and Pat, a deaf/hard of hearing white woman.

\section{B. Analysis}

Our broad goal is to better understand the dynamics of in/equity in collaborative small groups. In past work investigating group dynamics we have found "inflection points" to be valuable places to look closely at, ie. [6]. By inflection point we mean a moment when the dynamics change in some way. We aim to identify and then analyze those points using a mixed quantitative-qualitative methods approach. Quantitative analysis allows us to analyze a (relatively) large amount of data in an effort to identify smaller sections of the data for detailed, qualitative analysis. In our quantitative analysis we are looking for shifts in the nature of the group activity, and then we zoom in on the periods when the shift occurs to try and understand how or why it happened. 
The group we focus on was together for six days of the program and we analyzed video data across the entire six days. To identify patterns of status and in/equity within the group we initially used talking time as an indicator of equity. Talking time is a commonly used marker of in/equity in analysis of small groups [i.e. 3,7]; the underlying assumption is more equal distribution of talk marks more equitable group interactions. For each day, we coded talking time for 5-7 five-minute-long video segments. With one exception the video for each day was comprised of 15 to 20 segments. On those days we sampled every third segment to code. One day had a very short small group activity and we coded all of the video segments on that day.

We also used proposal negotiation units (PNUs) to quantify emergent status hierarchies [8]. A PNU is the set of interactions that includes an initial verbal or nonverbal proposal concerning academic or social topics made by one group member, the intervening negotiation of that proposal bid, and the conclusion of the interaction that results in the ultimate uptake, ignoring, or rejection of the proposal bid. The uptake or rejection of a proposal bid can be tabulated as status promotions or demotions respectively. The sum of promotions and demotions indicates the relative status of an individual within the group (i.e. high status or low status). Because PNU analysis is a time intensive coding process - it requires creating a transcript of the video - we were selective in doing it. We chose days 4 and 6 , and selected continuous 25-minute segments on each day to code for PNUs. We chose those two days because they were significantly different from one another in terms of the distribution of talking time and we wanted to see if they looked different in the PNU analysis.

Our final analysis step involved creating a detailed qualitative description of group interaction at the beginning of day 4. As we will explain in the next section, day 4 was markedly different from the other days. The pattern of talking time distribution was consistent across all days with the exception of day 4. To better understand the negotiation of status and in/equity we analyzed transcript, body positioning, and movement of materials from the first 10 minutes of day 4 .

\section{RESULTS AND DISCUSSION}

From our analysis, a clear pattern of inequitable student interactions emerged. Fig. 1 shows the tabulated relative status of each group member on a typical day (day 6). On most days, Justin was the high-status member of the group while his peers were lower-status members of the group. Unfortunately, Justin's high status, which our tabulation of status promotions and demotions shows, appears to have afforded him the ability to dominate the group, which resulted in highly inequitable group dynamics. He spoke more often than any other group member, constantly tried to assert his intellectual and social authority over others, and often had his ideas and suggestions taken up by the group, even when the quality of his contributions was questionable.

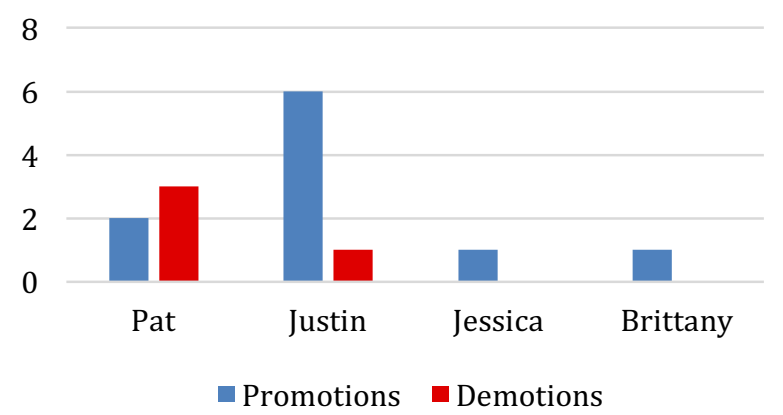

FIG 1. Tabulated status promotions (uptake of that student's proposal bid by the group) and demotions (rejection of that student's proposal bid by the group) on day 6 calculated from 25 minutes of group video segments.

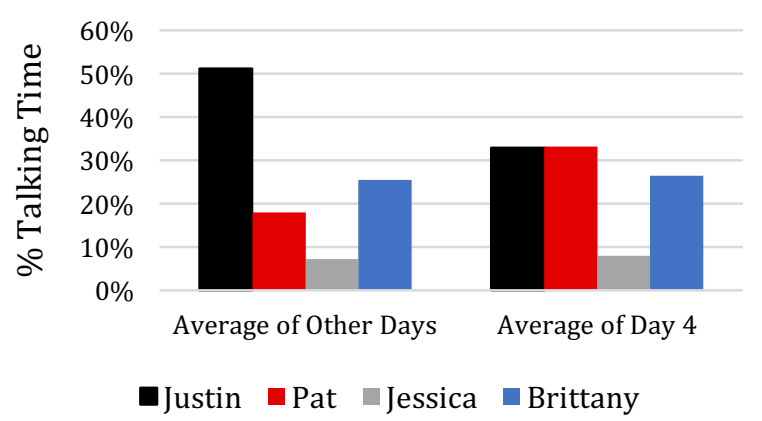

FIG 2. Average percent talking time on day 4 vs. other days. Percent talking time calculated by aggregating talking time. from 5-8 video segments per day. Each video segment was 5 minutes long.

While this pattern of inequity is apparent on most days, there is a notable deviation on day 4 . Talking time analysis shows that on days 1, 2, 3, 5, and 6 Justin dominates around $51 \%$ of the group's total talking time whereas on day 4 , Justin only accounts for $33 \%$ of talking time (Fig. 2). Another departure from the established pattern is the behavior of Pat, a member of the group who usually occupies a lower status. On most days, Pat's percent talking time is $18 \%$ whereas on day 4 , she increases to $33 \%$.

A closer qualitative analysis of day 4 reveals that there is a change in the status hierarchy of the group members on this day. It is apparent that the established student hierarchy is intact at the beginning of day 4 . The video shows Justin making numerous bids to dominate the conversation, often speaking over others and rejecting others' suggestions at the beginning of the day. The other group members attempt to reject and exclude Justin at various points, but he is persistent and eventually the other group members appear to accept Justin as a high-status member. This behavior is consistent 
with a larger pattern of Justin dominating group interactions that is consistently observed across days. However, the instructor markedly changes the group dynamic when he positions Pat as a group member with intellectual authority. The instructor does this by endorsing Pat's idea and

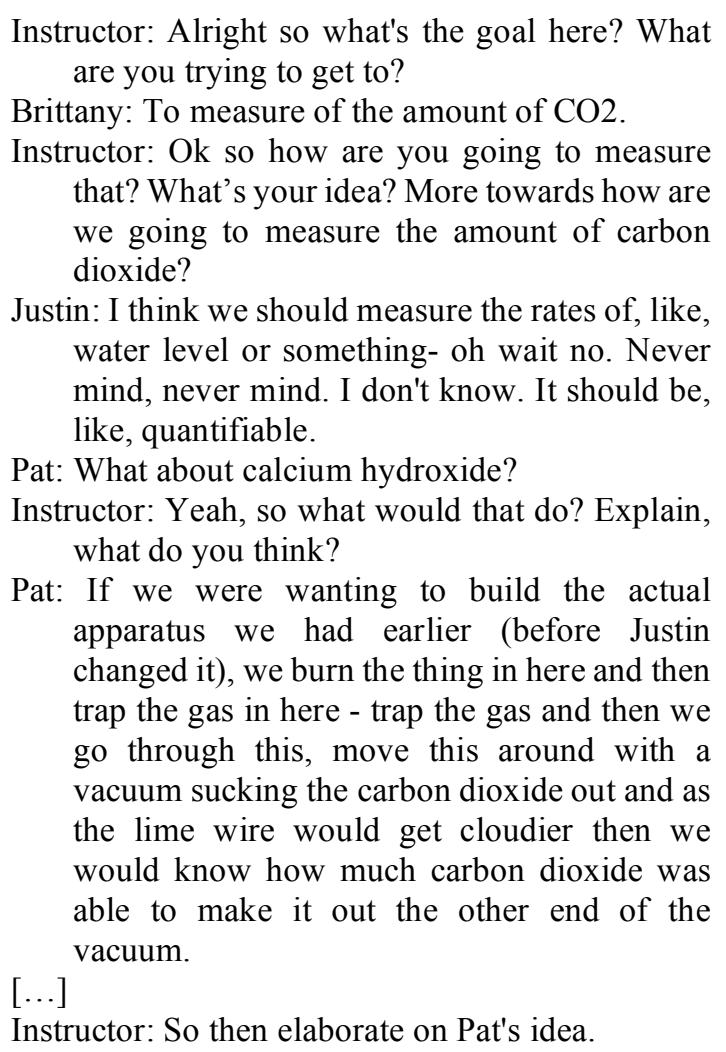
are you trying to get to?

Brittany: To measure of the amount of $\mathrm{CO} 2$.

Instructor: Ok so how are you going to measure that? What's your idea? More towards how are we going to measure the amount of carbon dioxide?

Justin: I think we should measure the rates of, like, water level or something- oh wait no. Never mind, never mind. I don't know. It should be, like, quantifiable.

Pat: What about calcium hydroxide?

Instructor: Yeah, so what would that do? Explain, what do you think?

Pat: If we were wanting to build the actual apparatus we had earlier (before Justin changed it), we burn the thing in here and then trap the gas in here - trap the gas and then we go through this, move this around with a vacuum sucking the carbon dioxide out and as the lime wire would get cloudier then we would know how much carbon dioxide was able to make it out the other end of the vacuum.

$[\ldots]$

Instructor: So then elaborate on Pat's idea.

FIG. 3 Transcript of teacher intervention from day 4. Students were tasked with designing an apparatus to measure the amount of $\mathrm{CO}^{2}$ produced from a combustion reaction.

directing the other group members to expand on it (see Fig. 3 for transcript of the episode). Following this short interaction, Pat began participating more and taking a greater leadership role in the activity. It appears Justin's influence over the group waned and he began contributing less.

Our PNU analysis of day 4 reflects the new status hierarchy after the instructor intervention. Fig. 4 shows our status tabulation based on the PNU analysis. It appears Pat has come to occupy a relatively higher status position while Justin is demoted in the social hierarchy. All of Pat's bids are taken up by the group, while Justin makes fewer bids than on the other day we analyzed (Fig. 1) and on this day most of his bids are rejected or ignored. Note the contrast of Pat on those two days as well. She makes a significant number of bids on both days, but on day 6 the majority of her bids are rejected or ignored.

Fig. 5 is particularly interesting because it shows the change on day 4 over time. In video segment 2 , prior to the instructor interventions, the distribution of talking time is similar to the aggregate of other days. Justin dominates, accounting for about $60 \%$ of the talk, with Brittany around $25 \%$ and Pat and Jessica around 10\% each. The instructor intervention occurs during segment 3, and we see Pat's percentage go up a bit in that segment and remain at that level in segment four. Meanwhile, Justin's contributions gradually decrease in segments 3 and 4. Then, in segment 5, Pat's contributions rise dramatically, approaching 60\%, while Justin's continue to drop to about $15 \%$. The other two group members, Brittany and Jessica, remain fairly constant across these segments. The talking time percentages in figure 5 pretty closely mirror our qualitative analysis; as Pat's status increases as a result of the instructor intervention, so does her talking time.

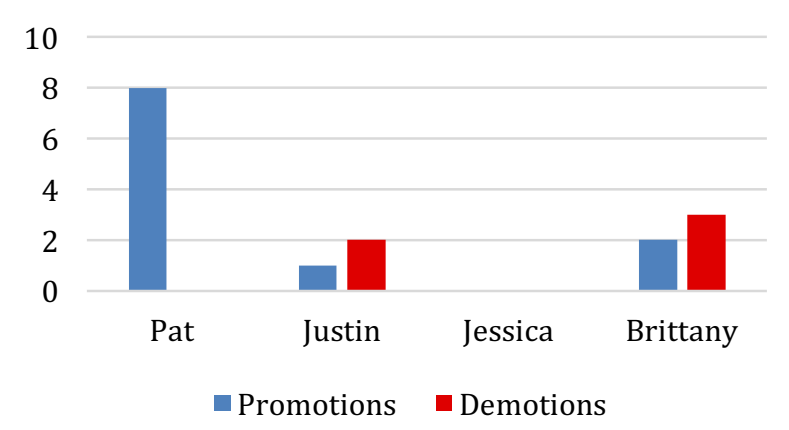

FIG 4. Tabulated status promotions and demotions on day 4 calculated from 25 minutes of group video segments.

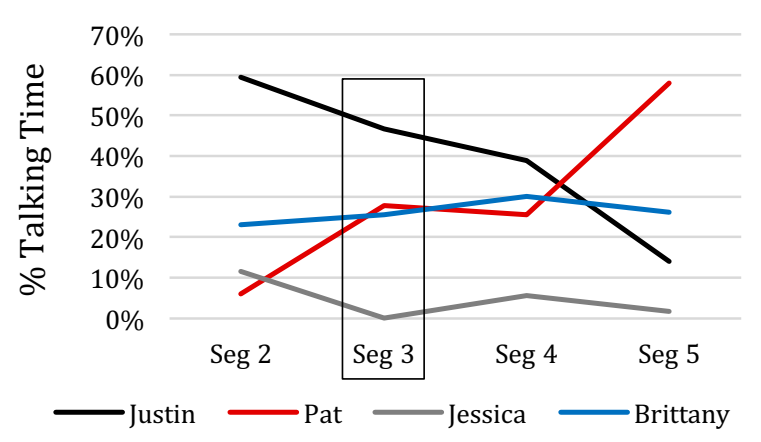

FIG 5. Percent talking time on day 4 calculated from four video segments. Each video segment was 5 minutes long. Rectangle represents the teacher intervention that occurs in segment 3 .

It is both interesting and somewhat disappointing that the group dynamics on the days after day 4 return to the pattern of inequity we observe in days 1 through 3 . On days 5 and 6 Justin again occupies the highest-status position and dominates group interactions, while Pat, Brittany, and Jessica are lower status group members who are often unable to participate due to Justin's domineering behavior.

The instructor intervention shown in Fig. 3 appears to be a rather routine check in to make sure the group is starting 
off the task in a productive manner. Our analysis suggests that the fact the instructor endorsed Pat's idea resulted in a disruption of the pretty firmly established status dynamics of this group and that disruption persisted through the entire activity of that day. It did not, however, carry over to the following days.

\section{CONCLUSIONS}

The purpose of this study was to investigate the relationship between social positioning of students and the in/equitable distribution of learning opportunities in cooperative learning groups in collegiate science education. While this study analyzes only a single case study, this work contributes to a larger literature on status hierarchies, equity, and the importance of teacher interventions. We examined one group of students that exhibited a clear and consistent pattern of inequitable interaction. This inequity occurred as a result of an established status hierarchy that positioned one student, Justin, as being more competent and authoritative in comparison to his peers. His high status gave Justin greater access to learning opportunities and allowed him to limit his peers' participation, and thereby limited their learning opportunities. While this study was not intended to examine gender dynamics in science education, it would be remiss not to note that Justin's domination of the group is interesting considering that he was the only man the group. This pattern of interaction mirrors broader patterns of marginalization of women in the field of science as a whole.

The only deviation from the pattern of inequitable interactions occurred when an instructor successfully disrupted the established status hierarchy of the group. A short conversation in which the instructor endorses Pat's idea and encourages the group to expand upon it is immediately followed by a disruption to the status hierarchy of the group. With the available data it is difficult to claim with certainty that the change in social hierarchy was a result of the teacher intervention, but because this is the only discernable abnormality that precedes the change in group interaction, it seems fairly likely. This change in the social organization of the students resulted in more equitable group interaction, supporting the idea that social hierarchies can contribute to inequity in student groups. The profound change in the quality of student interaction that occurred seemingly as a result of the teacher intervention suggests that instructor feedback can significantly affect the social organization of students. Other research has similarly found that teacher evaluations of student ideas and behavior can have lasting effects on subsequent student interactions. [9] This has meaningful implications for educators, who have the potential to intervene in inequitable student interactions in order to support productive and equitable student collaborations.

While the reorganization of the group's status hierarchy and the associated change in group dynamics persisted for the remainder of day 4 , the group returned to the previously established pattern of inequitable interaction on subsequent days. This suggests that while instructor interventions can have a profound effect on student interaction, these changes to student group dynamics are not permanent and without continued instructor mediation they can revert back to less productive patterns of interaction.

The analysis presented here suggest various avenues for future research on the complex relationship between student status hierarchies, equity, and the role of the instructor in cooperative learning. The negotiation of students' status and relative positioning within a group is a very complicated process that it not yet fully understood. Additional research is necessary to determine how students come to create status hierarchies in the classroom. This case study also illustrates the important role of the instructor in shaping student interactions. Further research is necessary to determine how instructors can best intervene to promote student equity and foster productive student collaborations. Additionally, because the effects of instructor interventions do not appear to be permanent, a productive avenue for further research would be determining how to make more lasting changes to student interactions.

\section{ACKNOWLEDGEMENTS}

The authors wish to thank the Rochester Institute of Technology and the PEER program for making this research possible. We also thank Grinnell College for their support.
[1] Y. Xie, M. Fang, and K. Shauman, Annual Review of Sociology, 41, 331-357 (2015).

[2] I. Esmonde, Journal of the Learning Sciences, 18, 2 (2009).

[3] C.M. Lewis and N. Shah, Proceedings of the eleventh annual International Conference on International Computing Education Research, 41-50 (2015).

[4] J.M. Langer-Osuna, Canadian Journal of Science, Mathematics and Technology Education, 11, 3 (2011).

[5] S. Franklin, E. Hane, and C. Ptak, (in preparation).
[6] P. Hutchison and D. Hammer, Science Education, 94, 3 506-524 (2010)

[7] E. Deitrick, R. Shapiro, and B. Gravel, Proceedings of the $12^{\text {th }}$ International Conference of the Learning Sciences (2016).

[8] R.A. Engle, J. M. Langer-Osuna, and M. McKinney de Royston, Journal of the Learning Science, 23, 2, 245268 (2014).

[9] J. M. Langer-Osuna, Mathematical Thinking and Learning, 18, 2, 107-124 (201 